\title{
Constraints on ultraviolet-stable fixed points in supersymmetric gauge theories
}

\author{
Stephen P. Martin ${ }^{a}$ and James D. Wells ${ }^{b}$ \\ ${ }^{(a)}$ Department of Physics, Northern Illinois University, DeKalb IL 60115 and \\ Fermi National Accelerator Laboratory, PO Box 500, Batavia IL 60510 \\ ${ }^{(b)}$ Physics Department, University of California, Davis CA 95616, and \\ Theory Group, Lawrence Berkeley National Laboratory, Berkeley CA 94720
}

\begin{abstract}
We consider the possibility that some supersymmetric gauge theories which are not asymptotically free can be governed by ultraviolet-stable fixed points. If this scenario can be realized, gaugino masses will exhibit power-law running with scale, providing a possible solution to the supersymmetric flavor problem. While naive perturbative calculations hint at the appearance of ultraviolet-stable fixed points in certain theories, there are strong constraints following from limits on the scaling dimensions of gauge-invariant operators, positivity of central charges, and Cardy's conjectured constraint on the flow of the Euler coefficient in the stress tensor trace anomaly. Also, we prove that if ultraviolet-stable fixed points do exist, they cannot occur in the perturbative regime of a renormalizable model, and that all-orders results in the limit of large numbers of chiral superfields are necessarily inconclusive. However, we argue that the general idea of ultraviolet-stable fixed points in supersymmetric gauge theories is viable, and exhibit models that can satisfy all known constraints.
\end{abstract}

PACS numbers: 11.30.Pb, 12.60.Jv, 11.10.Hi

hep-ph/0011382, FERMILAB-Pub-00/301-T, LBNL-47122

\section{INTRODUCTION}

Our understanding of the high-energy behavior of gauge couplings in supersymmetric (SUSY) theories depends crucially on the matter content of the model. If the number of chiral superfields is sufficiently small, the oneloop beta function for gauge couplings is negative, and the ultraviolet (UV) theory is formally governed by a free fixed point in which the renormalizable gauge couplings are driven to zero.

In constrast, it is usually assumed that supersymmetric gauge theories with positive one-loop $\beta$ functions have a Landau pole in the UV. The scale of the Landau pole may represent the energy above which new physics must enter in the form of e.g. extra dimensions, string interactions, or quantum gravity. Above that scale, either the running of the gauge coupling is modified so that it remains finite, or else the gauge coupling has no simple meaning within the new framework in the far UV. In either case, it is quite difficult to understand in detail how to match the model to a more fundamental theory. There are many otherwise-attractive extensions of the minimal supersymmetric standard models (MSSM) [1] that seem to fit into this category. For example, the one-loop beta functions are large and positive in many supersymmetric grand unified theories (GUTs) and in string-inspired models with large numbers of vectorlike representations.

In this paper, we will consider another possibility, the idea that a renormalizable supersymmetric gauge theory may have a non-trivial UV-stable fixed point associated with a theory with exact or approximate superconformal invariance. In this scenario, the UV theory approaches a critical point in which (at least some) supersymmetric gauge and Yukawa couplings are nearly stationary, and soft supersymmetry-breaking couplings have a powerlaw running with scale. The criticality may be only approximate, if some couplings are small. What differentiates this from the situation in the previous paragraph is that the theory can remain near the critical point for a significant range of energy scales up to the Planck scale (or another scale of fundamental physics such as a compactification scale or string scale).

If a UV fixed point can indeed be realized, it has interesting and unique implications. For example, we will show below that gaugino masses necessarily scale to zero by a power law in the UV. This enables large hierarchies in soft masses to be generated from renormalization group (RG) running. These hierarchies could be used to suppress flavor-violating interactions in supersymmetry. Another intriguing feature of the UV fixed point idea is that it could be related to the apparent need for semi-perturbative unification in string theory [2, 3]. In string theory with weak coupling, the dilaton potential has no stable vacuum. Strongly-coupled string theories naively have the same problem since they are dual to weakly-coupled theories. Perhaps an intermediatestrength coupling of a settled and non-trivial $(g \neq 0) \mathrm{UV}$ fixed point could be part of the solution to this problem.

However, it is not clear whether renormalizable supersymmetric models with UV-stable fixed points actually exist in four dimensions. As we will show, there are some hints from finite-order calculations that weakly suggest such UV-stable fixed points can occur. However, there 
are also strong constraints that follow from the general properties of superconformal theories, from the fact that scaling dimensions of gauge-invariant operators cannot be less than 1, from the positivity of central charges, and from the irreversibility of renormalization group RG flows as embodied in Cardy's conjecture [4] generalizing to 4 dimensions the Zamolodchikov $c$-theorem in 2 dimensions [5]. We will examine these restrictions, and show that there are some simple models that can satisfy all known constraints including Cardy's conjecture.

\section{THE NSVZ $\beta$ FUNCTION, ANOMALOUS DIMENSIONS, AND R-CHARGES AT SUPERCONFORMAL FIXED POINTS}

We begin by discussing some general properties that must be satisfied by fixed points in a supersymmetric gauge theory. The existence of a fixed point should be independent of the renormalization scheme used. However, it is most convenient to use the NSVZ scheme, since there is an exact relation between beta functions and the anomalous dimensions and $R$-charges of chiral superfields. In our notation, the NSVZ gauge coupling $\beta$ function is given by

$$
\beta(g)=\frac{g^{3}}{16 \pi^{2}}\left[\frac{-3 C_{G}+\sum_{r} I_{r}\left(1-2 \gamma_{r}\right)}{1-C_{G} g^{2} / 8 \pi^{2}}\right],
$$

where $C_{G}$ is the Dynkin index of the adjoint representation, the sum $\sum_{r}$ is over the irreducible representations of the chiral superfields with chiral superfield anomalous dimensions $\gamma_{r}$, and $I_{r}$ is the Dynkin index of the representation $r$. The normalizations are such that $C_{G}=N_{c}$ and $I_{r}=1$ for each fundamental plus anti-fundamental representation of $S U\left(N_{c}\right)$. The $\beta$ function has a pole at very strong coupling: $g_{\text {pole }}^{2}=8 \pi^{2} / C_{G}$, but we assume that this plays no role and any fixed points occur at weaker coupling $g_{*}<g_{\text {pole }}$. The numerator of $\beta(g)$ must therefore vanish at a fixed point $g=g_{*}$ corresponding to a scale-invariant theory.

Although there is no proof for four dimensions, one expects the equivalence of scale invariant theories and conformal theories [6]. The conformal symmetries are necessarily unified with supersymmetry transformations to form the superconformal algebra. This implies stringent constraints, since the superconformal symmetry includes a non-anomalous $U(1) R$-symmetry and an invariance under scaling. The $R$-charge and superconformal scaling dimension $D$ for any gauge-invariant chiral superfield must be related by [7]

$$
D=3 R / 2 \text {. }
$$

Since the scaling dimension for any composite chiral superfield is the sum of $1+\gamma_{r}$ for its constituents, it follows that

$$
3\left(R_{r}-1\right)=2 \gamma_{r}\left(g_{*}\right)-1
$$

at a superconformal fixed point $g=g_{*}$. This establishes a connection between the conditions for the vanishing of the NSVZ $\beta$ function and the cancellation of the $R$ symmetry anomaly. In particular, since the $R$-charge of the chiral fermion in the representation labelled by $r$ is $R_{r}-1$, and that of the gaugino is 1 , the anomaly cancellation requirement

$$
C_{G}+\sum_{r} I_{r}\left(R_{r}-1\right)=0
$$

is equivalent to the vanishing of the numerator in eq. (11). In some theories, where either symmetries or the presence of a superpotential are sufficient to fix the $R$-charges uniquely, this allows an exact non-perturbative determination of the anomalous dimensions at the fixed point. More generally, it gives one linear relation between the anomalous dimensions, if the assignment of $R$-charges is not uniquely determined. Therefore, eq. (2) is essentially tautological (provided that the appropriate $R$-symmetry can be uniquely determined) rather than a non-trivial constraint in superconformal theories.

If $\beta\left(g_{*}\right)=0$ and $\beta(g)<0$ for $0<g<g_{*}$, then $g_{*}$ can be an IR-stable fixed point. For example, in supersymmetric QCD with $N_{c}$ colors and $N_{f}$ flavors of quark and antiquark superfields, it has been argued 8,9] that there is an IR-stable fixed point for $3 N_{c} / 2<N_{f}<3 N_{c}$. The $R$-charges of the quark and antiquark superfields are both equal to $R_{Q}=R_{\bar{Q}}=$ $1-N_{c} / N_{f}$ corresponding to an anomalous dimension (in our normalization) $\gamma_{Q}\left(g_{*}\right)=\gamma_{\bar{Q}}\left(g_{*}\right)=\left(1-3 N_{c} / N_{f}\right) / 2$. While the existence of the IR-stable fixed points in the theories is conjectured, the relations can be checked in perturbation theory when $3 N_{c}-N_{f}$ is very small, as in the case of non-supersymmetric QCD 10]. A conjectured dual description can be used to check the relations when $N_{f}-3 N_{c} / 2$ is small and positive. This lends credence to the picture of a pair of interacting superconformal theories having the same physics in the IR. Other examples include deformations of $N=2$ and finite $N=1$ theories, which generally can have IR-stable fixed lines [11].

If instead $\beta\left(g_{*}\right)=0$ and $\beta(g)>0$ for the range $0<g<g_{*}$, then $g_{*}$ is a UV-stable fixed point. This is the case of interest in this paper. Note that this implies $\partial \beta / \partial g<0$ at the fixed point, a fact that will be important in the following. It also requires that $\sum_{r} I_{r} \geq$ $3 C_{G}$ so that the leading-order $\beta$ function is positive. The question then becomes whether theories that satisfy this can be found with anomalous dimensions and $R$ charges satisfying eqs. (3) and (4). Before addressing the specific constraints on this scenario, it is convenient to first consider what we can say about the RG running 
of soft supersymmetry-breaking parameters in a realistic version of such a model.

\section{EVOLUTION OF SOFT SUPERSYMMETRY-BREAKING PARAMETERS NEAR A UV-STABLE FIXED POINT}

In this section, we assume for the moment that the exact $\beta$ functions of the theory do admit a UV-stable fixed point. In a realistic model, the superconformal invariance will not be exact. Let us assume that SUSY breaking is communicated, for example by supergravity mediation, to the MSSM sector at a high scale $Q_{0}$ within the regime of the fixed point. In general, the soft supersymmetry breaking mass parameters will undergo power-law RG evolution, so that in the limit $Q \ll Q_{0}$,

$$
m_{\text {soft }}(Q) \sim\left(Q_{0} / Q\right)^{k}
$$

where $k$ is a critical exponent for the particular soft mass, and $Q$ is the RG scale. If $k>0$, then the corresponding soft term grows as we move towards the IR within the fixed point region. In general, we would like to investigate the properties of the critical scaling for soft terms in realistic models.

For simplicity, we begin with the case of a model with a simple gauge group and superpotential couplings sufficiently small that they can be neglected. Remarkably, the exact gaugino mass beta function is related [12] to the exact gauge coupling beta function in the NSVZ scheme by the simple expression

$$
\beta_{M}=2 M g^{2} \frac{\partial}{\partial g^{2}}(\beta / g)
$$

At a UV-stable fixed point, $\beta=0$ and $\partial \beta / \partial g<0$ must hold. It follows that

$$
\frac{d M}{d t}=\beta_{M}=-\kappa M
$$

where $t=\ln Q$ and

$$
\kappa \equiv-\partial \beta / \partial g
$$

is a positive constant in the fixed point regime, defined as $g$ for which $|\partial \beta / \partial g| \gg|\beta / g|$. Note that $\kappa$ is independent of the choice of renormalization scheme.

The scale dependence of the gaugino mass is

$$
M(Q)=\left(Q_{0} / Q\right)^{\kappa} M_{0}
$$

Thus $M$ runs according to a power law in the fixed point region. If $Q_{0}$ is the boundary condition scale where supersymmetry breaking is transmitted, then it is possible to have $M_{0}$ at this high scale be very small, yet induce large $M$ at lower scales $Q$ due to the power law running in the fixed point region. The gravitino mass, on the other hand, is presumably correlated with the boundary condition gaugino mass, $m_{3 / 2} \sim M_{0}$. Therefore, the physical gaugino can be significantly more massive than the gravitino. The ratio of the masses is estimated as

$$
M / m_{3 / 2} \sim\left(Q_{0} / Q_{L}\right)^{\kappa}
$$

where $Q_{L}$ is the lowest scale of the UV fixed point regime.

The above results can be generalized to more complicated models. As we will see in section VIII, it is quite possible that the existence of a UV-stable fixed point requires the presence of superpotential couplings. If the ultimate gauge group is not simple, then it is possible that several gauge couplings will be significant at the fixed point. Therefore, let us consider the more general possibility of a fixed point with several distinct non-zero gauge couplings $g_{A}$ and non-zero holomorphic Yukawa couplings $Y_{i j k}$. The superpotential is

$$
W=\frac{1}{6} Y^{i j k} \Phi_{i} \Phi_{j} \Phi_{k}
$$

Now consider the RG running of deviations from the fixed point: $g_{A}=g_{A *}+\delta_{A} ; Y^{i j k}=Y_{*}^{i j k}+\delta^{i j k} ; Y_{i j k}=Y_{i j k *}+$ $\delta_{i j k}$. One obtains:

$$
\frac{d}{d t}\left(\begin{array}{c}
\delta_{A} \\
\delta^{i j k} \\
\delta_{i j k}
\end{array}\right)=-C\left(\begin{array}{c}
\delta_{B} \\
\delta^{l m n} \\
\delta_{l m n}
\end{array}\right)
$$

where

$$
C=-\left(\begin{array}{ccc}
\partial \beta_{A} / \partial g_{B} & \partial \beta_{A} / \partial Y^{l m n} & \partial \beta_{A} / \partial Y_{l m n} \\
\partial \beta^{i j k} / \partial g_{B} & \partial \beta^{i j k} / \partial Y^{l m n} & \partial \beta^{i j k} / \partial Y_{l m n} \\
\partial \beta_{i j k} / \partial g_{B} & \partial \beta_{i j k} / \partial Y^{l m n} & \partial \beta_{i j k} / \partial Y_{l m n}
\end{array}\right)
$$

Here we have used a notation in which $\beta_{A}$, $\beta^{i j k}$ and $\beta_{i j k}$ denote the $\beta$ functions of $g_{A}, Y^{i j k}$, and $Y_{i j k}$ respectively. Following common practice, complex conjugation of couplings is denoted by raising and lowering indices, e.g. $Y_{i j k}=\left(Y^{i j k}\right)^{*}$. By definition, at a UV-stable fixed point, all eigenvalues of $C$ must have non-negative real parts. For a realistic model, we assume that the superconformal invariance is only approximate, and in particular is violated by soft gaugino masses $M_{A}$ and by trilinear scalar couplings:

$$
\mathcal{L}=-\frac{1}{6} a^{i j k} \phi_{i} \phi_{j} \phi_{k}+\text { c.c. }
$$

The running of the soft gaugino masses $M_{A}$ and the trilinear couplings $a^{i j k}$ is then given exactly in terms of 
the NSVZ $\beta$ functions of the supersymmetric parameters by 12

$$
\frac{d}{d t}\left(\begin{array}{c}
g_{A} M_{A} \\
a^{i j k}
\end{array}\right)=-K\left(\begin{array}{c}
g_{B} M_{B} \\
a^{l m n}
\end{array}\right)
$$

where

$$
K=\left(\begin{array}{cc}
-\partial \beta_{A} / \partial g_{B} & 2 \partial \beta_{A} / \partial Y^{l m n} \\
\partial \beta^{i j k} / \partial g_{B} & -2 \partial \beta^{i j k} / \partial Y^{l m n}
\end{array}\right) .
$$

Here we have dropped terms which vanish at the fixed point.f The solution to this equation will have the form

$$
\left(\begin{array}{c}
g_{A} M_{A}(Q) \\
a^{i j k}(Q)
\end{array}\right)=\sum_{n}\left(Q_{0} / Q\right)^{\kappa_{n}} \mathcal{M}^{(n)}
$$

where $\kappa_{n}$ are eigenvalues of $K$ corresponding to eigenvectors

$$
\mathcal{M}^{(n)}=\left(\begin{array}{c}
\mathcal{M}_{A}^{(n)} \\
\mathcal{M}^{(n) i j k}
\end{array}\right)
$$

In general the eigenvalues of $C$ do not correspond to eigenvalues of $K$. However, the traces of the matrices $C$ and $K$ do have the same real part. Therefore, at least one of the eigenvalues $\kappa_{n}$ must have a positive real part, since the sum of the eigenvalues of a matrix is equal to its trace. This proves that the running of the gaugino masses and the trilinear scalar couplings is again governed in the limit $Q \ll Q_{0}$ by a negative critical exponent, corresponding to the eigenvector of $K$ with the eigenvalue with the largest real part. This again implies power-law growth of all soft terms as one moves towards the IR within the fixed-point region.

The behavior of soft scalar squared mass RG evolution is more difficult to ascertain. We can still obtain some insight, however, by considering the form of the RG equations near the fixed point. We will begin by considering this in the case of a general model with arbitrary gauge and Yukawa couplings, and then specialize to the simpler case where Yukawa couplings vanish (or can be safely neglected) at the fixed point. Even in the case of a general model, the IR values of the subset of soft squared masses of scalars which do not have large Yukawa couplings at the UV-stable fixed point will depend only on their gauge quantum numbers, providing a possible solution to the supersymmetric flavor problem in the MSSM.

The scalar squared mass terms are

\footnotetext{
${ }^{\dagger}$ Actually, eq. (16) need not be valid for $i, j, k$ with vanishing $Y^{i j k}$. However, the corresponding $a^{i j k}$ have homogeneous $\mathrm{RG}$ equations, and do not contribute to the $\mathrm{RG}$ equations for gaugino masses, so they decouple from this discussion.
}

$$
\mathcal{L}=-\left(m^{2}\right)_{i}^{j} \phi^{* i} \phi_{j}
$$

The RG equations for $\left(m^{2}\right)_{i}^{j}$ contain inhomogeneous terms quadratic in gaugino masses and $a^{i j k}$ terms, and homogeneous terms. Therefore, we can write

$$
\frac{d}{d t}\left(m^{2}\right)_{i}^{j}=\sum_{n, m}\left(Q_{0} / Q\right)^{\kappa_{n}+\kappa_{m}^{*}}\left[\Gamma_{n, m}\right]_{i}^{j}-L_{i l}^{j k}\left(m^{2}\right)_{k}^{l} .
$$

The fixed-point quantities $\left[\Gamma_{n, m}\right]_{i}^{j}$ and $L_{i l}^{j k}$ can be given exactly in terms of first and second derivatives of the chiral superfield anomalous dimension matrix $\gamma_{i}^{j}$ with respect to the couplings $g_{A}, Y^{i j k}$ and $Y_{i j k}$, using the results of [14].

The general solution to eq. (20) takes the form

$$
\begin{aligned}
\left(m^{2}\right)_{i}^{j}= & \sum_{n, m}\left(Q_{0} / Q\right)^{\kappa_{n}+\kappa_{m}^{*}}\left[X_{n, m}\right]_{i}^{j} \\
& +\sum_{N}\left(Q_{0} / Q\right)^{\lambda_{N}}\left[X_{N}\right]_{i}^{j},
\end{aligned}
$$

where $\lambda_{N}$ and $\left[X_{N}\right]_{i}^{j}$ are eigenvalues and eigenvectors of the matrix $L_{i l}^{j k}$, in the sense

$$
L_{i l}^{j k}\left[X_{N}\right]_{k}^{l}=\lambda_{N}\left[X_{N}\right]_{i}^{j},
$$

and $\left[X_{n, m}\right]_{i}^{j}$ are other constants fixed by the boundary conditions.

As one possible solution to the supersymmetric flavor problem, suppose that a subset of the scalar fields including the squarks and sleptons of the MSSM do not have large Yukawa couplings participating in the fixed point. The contributions to the RG eq. (20) for the corresponding scalar squared masses are then clearly flavor-independent. In the idealized limit of a long running near the fixed point, $Q \ll Q_{0}$, one can write solutions in terms of $\kappa$ (the largest of the $\operatorname{Re}\left[\kappa_{n}\right]$ ) and $\lambda$ (the largest of the $\operatorname{Re}\left[\lambda_{N}\right]$ ). For the flavor diagonal scalar squared masses they will be of the form:

$$
\begin{aligned}
m_{i}^{2}(Q)= & x_{i} \bar{M}_{0}^{2}\left(Q_{0} / Q\right)^{2 \kappa} \\
& +\left(y_{i} \bar{m}_{0}^{2}-x_{i} \bar{M}_{0}^{2}\right)\left(Q_{0} / Q\right)^{\lambda} .
\end{aligned}
$$

Here $x_{i}$ and $y_{i}$ are constants related to the eigenvectors of the matrices $K,\left[\Gamma_{n, m}\right]_{i}^{j}$ and $L_{i l}^{j k}$, while $\bar{M}_{0}^{2}$ and $\bar{m}_{0}^{2}$ denote the overall scale of the boundary conditions for holomorphic and non-holomorphic supersymmetry breaking terms, respectively, at the scale $Q_{0}$.

By inspection of eq. (23), there are two ways to have positive and flavor-conserving soft scalar squared masses in the infrared:

$$
\text { - } 2 \kappa>\lambda \text { and } x_{i} M_{0}^{2}>0 \text {, or }
$$




$$
\text { - } \lambda>2 \kappa \text { and } y_{i} \bar{m}_{0}^{2}-x_{i} \bar{M}_{0}^{2}>0 .
$$

Since the relevant quantities depend on the second derivatives of anomalous dimensions at the fixed point, we do not see any reliable way to estimate them, or even determine their signs, far from the realm of perturbation theory, in a completely general theory. However, we do know that at least one $\operatorname{Re}\left[\kappa_{n}\right]$ is positive near a UV-stable fixed point.

If we further specialize to the case that there is only one large gauge coupling and no large Yukawa couplings at all at the fixed point, then the anomalous dimension matrix and derivatives of it are simultaneously diagonal, and one has from [14 the exact formula:

$$
L_{i l}^{j k}=\frac{g^{3} / 8 \pi^{2}}{\left(1-g^{2} C_{G} / 8 \pi^{2}\right)} \frac{\partial \gamma_{i}}{\partial g} \frac{C(k)}{d_{G}} \delta_{i}^{j} \delta_{l}^{k},
$$

where $d_{G}$ is the dimension of the adjoint representation. It follows that all of the $\lambda_{N}$ vanish except for a single eigenvalue, which can be evaluated as

$$
\lambda=-\partial \beta / \partial g=\kappa .
$$

This implies that the first of the above two scenarios is the appropriate one, with the soft terms at low scale being dominated by the gaugino mass contributions which overwhelm any flavor-violating non-holomorphic contributions. One also finds for the coefficient of $\left(Q_{0} / Q\right)^{2 \kappa}$ in the first term in eq. (20) the exact result

$$
\Gamma_{i}^{j}=\frac{g}{2}\left|M_{0}\right|^{2}\left[g \frac{\partial^{2} \gamma_{i}}{\partial g^{2}}+\left(\frac{3-g^{2} C_{G} / 8 \pi^{2}}{1-g^{2} C_{G} / 8 \pi^{2}}\right) \frac{\partial \gamma_{i}}{\partial g}\right] \delta_{i}^{j},
$$

where $M_{0}$ is the gaugino mass at $Q_{0}$. In order to have a positive $m_{i}^{2}$ resulting in the IR from the fixed-point running, it is necessary that the quantity in brackets in eq. (26) is negative for the corresponding scalar field labelled by $i$. However, with present methods we do not know how to evaluate the sign of this quantity even in the simplest models, because it involves the second derivative of the anomalous dimension.

Returning to the more general case with several relevant gauge groups and/or Yukawa couplings, the critical behavior may well still be dominated by gaugino contributions. In practice, several eigenvalues $\kappa_{n}$ may have real parts that are close together, so that the contributions from several eigenvectors need to be included. Also, there may be unknown non-perturbative corrections due to naively non-renormalizable terms in the Kähler potential which become relevant at the fixed point. In order to solve the supersymmetric flavor problem, however, a sufficient condition is merely that a critical exponent related to gaugino masses dominates the evolution of scalar squared masses near the fixed point for a large enough range of scales. Because the running is power-law rather than logarithmic, subdominant flavor-violating contributions can be overwhelmed.

\section{CONSTRAINT FROM THE CONFORMAL DIMENSION OF THE GAUGINO BILINEAR}

In any theory with conformal invariance (supersymmetric or not), unitarity requires that the scaling dimension of any gauge-invariant scalar operator $\phi$ must satisfy

$$
\Delta_{\phi} \geq 1
$$

with equality for a free field 13]. This gives strong contraints on possible fixed points. Before asking how this impacts on the possibility of a UV-stable fixed point in supersymmetry, we should note a slight subtlety. If $\phi$ is the scalar component of a chiral superfield $\Phi$, then it is not always true that the ordinary scaling dimension $\Delta_{\phi}$ is equal to the superconformal scaling dimension of the superfield, $D_{\Phi}$. For example, they will differ if $\Phi$ appears in the superpotential, as can be checked in the case of perturbative fixed points deformed by Yukawa couplings in the IR superconformal window of SUSY QCD. $D_{\Phi}$ participates in the essentially tautological rule of eq. (2), whereas $\Delta_{\phi}$ satisfies the non-trivial rule of eq. 27.)

Typically, eq. (27) will not yield a constraint for operators $\phi$ built out of chiral fermions and scalars at a UV-stable fixed point, since the anomalous dimensions are positive. However, in the case of a simple gauge group with no superpotential, the anomalous dimension of the gaugino bilinear field $\lambda \lambda$ is given by

$$
\gamma_{\lambda \lambda}=\beta_{M} / M=-\kappa,
$$

where the last equality only holds in the vicinity of the fixed point and follows from the previous section. Therefore the scaling dimension of the field is

$$
\Delta_{\lambda \lambda}=3-\kappa .
$$

Enforcing the constraint eq. (27), one finds

$$
\kappa \leq 2 \text {. }
$$

Thus there is a constraint on the slope of the $\beta$ function at the fixed point, corresponding also to a restriction on the critical exponent for the gaugino mass. [In the case of an IR-stable fixed point, $\kappa \leq 0$, so eq. (30) is automatically satisfied.] More generally, the same argument implies that all eigenvalues of the matrix $K$ of eq. (16) satisfy

$$
\operatorname{Re}\left[\kappa_{n}\right] \leq 2 .
$$

Note that $\lambda \lambda$ is also the lowest component of the chiral superfield whose $F$-term is the gauge field strength. The superconformal dimension $D$ of that chiral superfield is 3 , and its $R$-charge is 2 , as its $F$ component appears in the Lagrangian. This is consistent since, as we 
noted above, the ordinary conformal dimension $\Delta$ of the composite scalar field $\lambda \lambda$ can be and in fact is distinct from the superconformal scaling dimension $D$ of the chiral superfield to which it belongs.

We emphasize that in a realistic theory, the superconformal symmetry is actually broken by soft gaugino masses and small Yukawa and gauge couplings, but this breaking does not mean that the constraint eq. (30) does not apply. We can always imagine taking the realistic model and simply turning off the small superconformalbreaking effects; the constraint on the slope of the gaugecoupling $\beta$ function then applies to the supersymmetric couplings in that theory, and hence at least approximately to the realistic model.

\section{HINTS FROM PERTURBATIVE CALCULATIONS}

It is difficult to make a definitive statement about the existence of a UV fixed point even in SUSY theories, since this would require knowledge of the $\beta$ function at all loop orders in theories for which the one-loop contribution is positive. However, it is still worthwhile to examine the perturbative expansions of $\beta$ functions in theories which could have a UV-stable fixed point, as far as they are known. For simplicity, let us first consider a SUSY gauge theory with vanishing superpotential and a simple gauge group $G$. One can expand the gauge coupling $\beta$ function as

$$
\frac{d g}{d t}=\beta=g \sum_{n=1}^{\infty} b^{(n)}\left(\frac{g^{2}}{16 \pi^{2}}\right)^{n}
$$

The $b^{(n)}$ coefficients are scheme-dependent for $n \geq 3$, and are known [15] in the dimensional reduction (DRED) 16] and Novikov-Shifman-Vainshtein-Zakharov (NSVZ) 17] schemes to four-loop order. In the NSVZ scheme [15]:

$$
\begin{aligned}
b^{(1)}= & S_{0}-3 C_{G} \\
b^{(2)}= & 4 S_{1}+2 C_{G} S_{0}-6 C_{G}^{2} ; \\
b^{(3)}= & 4 C_{G}^{2} S_{0}+20 C_{G} S_{1}-12 C_{G}^{3}-8 S_{2}-4 S_{0} S_{1} ; \\
b^{(4)}= & 32 S_{3}+48(\zeta(3)-1) S_{1}^{2}-(8+48 \zeta(3)) C_{G} S_{0} S_{1} \\
& +8 C_{G}^{3} S_{0}+76 C_{G}^{2} S_{1}-40 C_{G} S_{2} \\
& -24 C_{G}^{4}+8 S_{0} S_{2}-4 S_{0}^{2} S_{1}
\end{aligned}
$$

where $S_{n} \equiv \sum_{r} I_{r} C_{r}^{n}$ and the sum $\sum_{r}$ is over all chiral superfield irreducible representations $r$. The normalizations are such that in $S U\left(N_{c}\right)$ with $N_{f}$ flavors of quark and anti-quarks,

$$
\begin{aligned}
C_{G} & =N_{c} ; \\
S_{n} & =N_{f}\left(\frac{N_{c}^{2}-1}{2 N_{c}}\right)^{n} .
\end{aligned}
$$

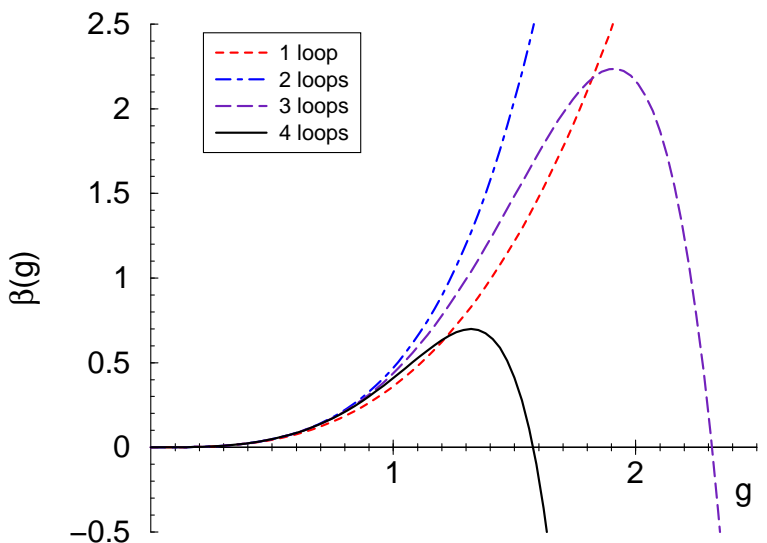

FIG. 1. The NSVZ gauge coupling $\beta$ function of the example $S U(5)$ minimal missing partner model at 1, 2, 3, and 4 loop orders.

In the DRED scheme, $b^{(1)}$ and $b^{(2)}$ are the same, but

$$
\begin{aligned}
b_{\mathrm{DRED}}^{(3)}= & 10 C_{G}^{2} S_{0}-21 C_{G}^{3}+26 C_{G} S_{1} \\
& -8 S_{2}-6 S_{0} S_{1}-C_{G}\left(S_{0}\right)^{2} ; \\
b_{\mathrm{DRED}}^{(4)}= & 32 S_{3}+48(\zeta(3)-1) S_{1}^{2}-102 C_{G}^{4} \\
& +54 C_{G}^{3} S_{0}+188 C_{G}^{2} S_{1}-80 C_{G} S_{2} \\
& -\left(\frac{184}{3}+48 \zeta(3)\right) C_{G} S_{0} S_{1}+\frac{64}{3} S_{0} S_{2} \\
& -\frac{14}{3} C_{G}^{2} S_{0}^{2}+\frac{4}{3} S_{0}^{2} S_{1}-\frac{2}{3} C_{G} S_{0}^{3} .
\end{aligned}
$$

From eqs. (33)-(36) we see that if $S_{0}>3 C_{G}$, then the one and two loop contributions to the $\beta$ function are positive. However, $b^{(3)}$ and $b^{(4)}$ can easily be negative, giving hope that the gauge coupling will reach a fixed point with $\beta=0$. In fact, this is quite often the case at both three- and four-loop order in both schemes.

As a potentially realistic example, consider the minimal missing partner $S U(5)$ model $[18]$, with $3 \times(\mathbf{5}+\overline{\mathbf{1 0}})$, $\mathbf{5}+\overline{\mathbf{5}}, \mathbf{2 4}, \mathbf{5 0}+\overline{\mathbf{5 0}}$ and $\mathbf{7 5}$ representations. The NSVZ $\beta$ functions at 1,2,3 and 4 loop orders are shown in fig. 1. We see that including the three- and four-loop terms, the $\beta$ function turns over and goes through zero. Note that the terms in the $\beta$ function are not just alternating in sign; both three- and four-loop contributions are negative. The three- and four-loop UV fixed points are quickly reached in RG running above the grand unified theory (GUT) scale $Q_{\mathrm{GUT}}$, so it is likely that the true UV fixed point will be reached (if it exists) significantly below the Planck scale.

Note that the negative slope of the $\beta$ function $\kappa=$ $-\partial \beta / \partial g$ obtained within the three- and four-loop approximations is too large to satisfy the bound eq. (30). Presumably, if this fixed point is indeed a feature of the full $\beta$ function, the approach to the fixed point must be appropriately smoothed.

The DRED scheme $\beta$ functions yield qualitatively 
similar results, although the locations of the fixed points are of course different. Also, we find that this behavior is quite generic in other GUT models with large representations, including those based on $S O(10)$. The same sort of thing happens as well in models with several gauge group factors and either large representations or large numbers of fundamental representations. For example, in supersymmetric QCD with $N_{f}$ flavors of quarks and antiquarks, it is not hard to show that the four-loop gauge $\beta$ function always has a zero for $N_{f} \geq 3 N_{c}$. The result for three-loop beta functions depends on the choice of NSVZ scheme or DRED scheme; in both cases a zero of the $\beta$ function requires that the number of flavors $N_{f}$ exceeds a critical value which can be obtained by solving a quadratic equation. In the NSVZ scheme, the critical value for $N_{f}$ is approximately $5 N_{c}$, while in the DRED scheme it is close to $4 N_{c}$.

It would be interesting (but of course hardly conclusive) to see whether this behavior continues at higher loop orders. Unfortunately, the calculation of $\beta$ functions at five or more loops seems to be beyond present technology. The so-called "exact" $\beta$ functions of SUSY Yang-Mills theories actually rely on knowledge of the anomalous dimensions of chiral superfields, which are only available to three-loop order except in special cases. In any case, no finite order calculation of the $\beta$ function could prove that a UV fixed point exists. Indeed, in the examples discussed here, the perturbative expansion of the $\beta$ function shows no signs of convergence in either the NSVZ or DRED schemes; the 1, 2, 3, and 4 loop contributions are all numerically of roughly the same order near the four-loop fixed point. However, the results above illustrate that it is possible for the full $\beta$ function to rise as $g$ does and then at some large value of $g$ to fall and cross zero, thereby implying a UV fixed point. Note that this does not involve any finetuning of parameters, but rather an assumption regarding the qualitative behavior of the full $\beta(g)$. The crucial question is simply whether the exact $\beta$ function behaves qualitatively like the 1 and 2 loop approximations in fig. 1, or like the 3 and 4 loop approximations. $\mathrm{f}$ We know of no existing argument or calculation that definitively answers this question.

\footnotetext{
${ }^{\ddagger}$ There is also a logical possibility that $\beta(g)$ remains nonzero for all $g$, but is small enough that $g$ does not diverge at any finite energy scale. This would correspond to a UV fixed point at infinite coupling. However, this seems unlikely in four-dimensional SUSY models because it would require the denominator of the NSVZ $\beta$ function to be conspiratorially cancelled.
}

\section{THE LARGE-INDEX LIMIT}

The results of the previous section suggest that we should probe the viability of UV-stable fixed points in the large- $N_{f}$ limit, or more generally in the limit of large Dynkin index for the chiral superfields, where some allorders results are now known. Bubble-sum calculations have been used to find the anomalous dimensions and gauge coupling $\beta$ functions for SUSY QCD with a large number of flavors $N_{f}$ in both the DRED and NSVZ schemes, to all orders in the loop expansion and to nextto-leading order in $1 / N_{f}$ [19]. These results can be written as a large-index limit in a general theory, in which only terms in $b^{(n)}$ with $n-1$ factors of $S_{i}$ are kept for $n \geq 2$. This calculation has a finite range of convergence $0 \leq g^{2}<24 \pi^{2} / S_{0}$ 19. In this section, we examine whether these results can say anything about UV-stable fixed points.

The results of [19] can be written in terms of a rescaled coupling:

$$
\hat{K} \equiv S_{0} g^{2} / 16 \pi^{2}
$$

and a special function

$$
G(x)=\frac{\Gamma(2-2 x)}{(\Gamma(1-x))^{2} \Gamma(2-x) \Gamma(1+x)} .
$$

Then, in a theory without Yukawa couplings, the anomalous dimension of each chiral superfield is [19]

$$
\gamma_{r}=-2 C_{r} \hat{K} G(\hat{K}) / S_{0}
$$

to the leading order in the large-index expansion. It follows that the NSVZ beta function is

$$
\beta^{\mathrm{NSVZ}}=g \hat{K}\left[\frac{1-3 C_{G} / S_{0}+4\left(S_{1} / S_{0}^{2}\right) \hat{K} G(\hat{K})}{1-2\left(C_{G} / S_{0}\right) \hat{K}}\right] .
$$

The DRED beta function is given by a more complicated expression [19]:

$$
\begin{aligned}
\beta^{\mathrm{DRED}}= & g \hat{K}\left[1-3 C_{G} / S_{0}+2\left(C_{G} / S_{0}\right) \int_{0}^{\hat{K}} G(x) d x\right. \\
& \left.+4\left(S_{1} / S_{0}\right) \int_{0}^{\hat{K}}(1-2 x) G(x) d x\right] .
\end{aligned}
$$

These results are exact to all-orders in $\hat{K}$, and to nextto-leading order in an expansion in $1 / S_{n}$. The interval

\footnotetext{
${ }^{\S}$ Note that our $g^{2}$ is equal to the $g^{2} / N_{f}$ of Ref. 19, our $\hat{K}$ is the same, and our $S_{0}$ assumes the role of $N_{f}$.
} 
of convergence for the infinite sum of bubble graphs is $0 \leq \hat{K}<3 / 2$, corresponding to a range over which $G(x)$ is finite.

In order to have a UV-stable fixed point, it is necessary to choose $S_{0}>3 C_{G}$ and to find a zero of the beta function. In the NSVZ case, this amounts to solving the equation

$$
\hat{K} G(\hat{K})=-S_{0}^{2} / 4 S_{1}
$$

where we consistently drop contributions higher order in $C_{G} / S_{0}$. This equation always has a solution for some $\hat{K}$ between 1 and $3 / 2$, since $G(x)$ is continuous and positive for $0 \leq x<1$ and $G(1)=0$ and $G(3 / 2)=-\infty$. This makes it appear that the large-index limit of the theory always has a UV-stable fixed point! However, it can also be shown without much difficulty that $\beta^{\text {DRED }}$ cannot have such a zero.

This apparent discrepancy between the two schemes is due to the fact that the putative UV-stable fixed point occurs in a regime in which the next-next-leading order corrections (of order $C_{G}^{2} / S_{0}^{2}$, etc.) are not negligible. Even though the sum to all orders in $\hat{K}$ is convergent, it is not trustworthy in the relevant region. Furthermore, in specific models one typically finds that the terms at 3 and 4 loop order which are the most negative do not show up at all in the large index (large $N_{f}$ ) expansion. Therefore, we must conclude that this expansion can make no unambiguous statement about the possibility of UV stable fixed points.

\section{UV STABLE FIXED POINTS CANNOT OCCUR WITH PERTURBATIVE COUPLINGS}

In the case of IR-stable fixed points, one can often tune the number of chiral fields to obtain arbitrarily small critical couplings, so that the existence of the fixed point can be reasonably established in some neighborhood within the space of models [10]. This is unfortunately not possible for UV-stable fixed points in supersymmetric theories. First consider the case in which there is no superpotential. In general, the existence of a perturbative fixed point requires that the one- and twoloop contributions in eq. (32) have opposite signs and $\left|b^{(1)}\right| \ll\left|b^{(2)}\right|$, so that

$$
\frac{g_{*}^{2}}{16 \pi^{2}} \approx-b^{(1)} / b^{(2)} \ll 1 .
$$

However, in supersymmetric theories the two-loop $g^{5} /\left(16 \pi^{2}\right)^{2}$ coefficient in the beta function is strictly larger than the one-loop $g^{3} / 16 \pi^{2}$ coefficient; $b^{(2)}=$ $b^{(1)}+4 S_{1}$. Therefore, if one tunes the one-loop coefficient to be either zero or small and positive, the two-loop contribution will necessarily be large and positive, since there is no way to tune $S_{1}$ to be small. This means that eq. (47) cannot be satisfied. Furthermore, it means that the location of a possible UV-stable fixed point cannot be made perturbative by balancing a large negative 3-loop contribution against controllably small one- and two-loop contributions.

It might seem that this argument could be evaded by introducing Yukawa couplings in a superpotential. Since Yukawa couplings enter at two loops into the gauge coupling $\beta$ function, this naively appears to allow the possibility of tuning both one- and two-loop to be small. However, the $\beta$ functions for those Yukawa couplings also must vanish at the fixed point. We can prove quite generally that this cannot happen with UV stability, as follows.

Consider a general superpotential of the form given in eq. (11). The $\beta$ function for the summed squares of all Yukawa couplings is

$$
\frac{d}{d t}\left(Y^{i j k} Y_{i j k}\right)=6 Y_{i j l} Y^{i j k} \gamma_{k}^{l}
$$

where $\gamma_{p}^{l}$ is the anomalous dimension matrix, and the indices $i, j, k, \ldots$ run over all chiral supermultiplet degrees of freedom. At one loop order, one has

$$
16 \pi^{2} \gamma_{l}^{(1) k}=\frac{1}{2} Y_{i j l} Y^{i j k}-2 \sum_{A} g_{A}^{2} C_{A}(k) \delta_{l}^{k}
$$

where the sum $\sum_{A}$ is over simple and $U(1)$ gauge groups, and $C_{A}(k)$ is the quadratic Casimir invariant of the chiral superfield carrying the index $l$. Requiring that the $\beta$ functions of the gauge couplings $g_{A}$ vanish, and rewriting eq. (48) as a sum over the irreducible representations of chiral superfields, chosen so as to diagonalize the matrix $\gamma_{l}^{(1) p}$ at the fixed point, one obtains to leading order:

$$
\frac{d}{d t}\left(Y^{i j k} Y_{i j k}\right)=12\left(16 \pi^{2} \sum_{r} d_{r}\left|\gamma_{r}^{(1)}\right|^{2}+\sum_{A} d_{A} b_{A}^{(1)} g_{A}^{2}\right)
$$

where $d_{A}$ is the dimension of the adjoint representation of the subgroup labelled by $A$, and the $\sum_{r}$ is over the irreducible representations of the chiral superfields with representation dimension $d_{r}$. At a fixed point, eq. (50) must vanish. This requires that some $b_{A}^{(1)}<0$ in order for a cancellation to occur on the right-hand side. So a perturbatively-reliable fixed point is only possible if a gauge-coupling $\beta$ function is significantly negative near weak coupling, implying an ultraviolet free theory or an unstable fixed point in the UV. We therefore conclude that stable UV fixed points cannot occur with perturbative couplings. 


\section{CONSTRAINTS FROM POSITIVITY OF CENTRAL CHARGES}

In this section we consider constraints following from the positivity of coefficients in the stress-energy trace anomaly. In an external supergravity background with sources for conserved flavor currents, the trace anomaly contains terms proportional to the square of the dual of the Riemann curvature, the square of the Weyl tensor, and the square of the flavor symmetry field strength. At a superconformal fixed point, the central charge coefficients for these terms can be shown to be respectively 20]:

$$
\begin{aligned}
a & =\frac{3}{32}\left(2 d_{G}+\operatorname{Tr}\left[\left(1-R_{i}\right)\left(1-3\left(1-R_{i}\right)^{2}\right)\right]\right) \\
c & =\frac{1}{32}\left(4 d_{G}+\operatorname{Tr}\left[\left(1-R_{i}\right)\left(5-9\left(1-R_{i}\right)^{2}\right)\right]\right) \\
b & =3 \operatorname{Tr}\left[\left(1-R_{i}\right) F_{i}^{2}\right] .
\end{aligned}
$$

Here the sums are over all chiral superfields with $R$ charges associated with the superconformal algebra $R_{i}$, flavor symmetry charges $F_{i}$, and $d_{G}$ is the dimension of the adjoint representation of the gauge group. At a non-interacting (free) fixed point, all of the $R_{i}$ should be replaced by $2 / 3$, and at an interacting fixed point they are constrained by the fact that the superconformal scaling dimension is $D=3 R / 2$ for gauge-invariant chiral superfields.

At a superconformal fixed point, each of the coefficients $a, b$, and $c$ must be positive 21,20]. In addition, Cardy's conjecture [4 implies that the generalization of Zamolodchikov's c-theorem in 2 dimensions should be that the coefficient $a$ is larger in the UV than in the IR. This expresses the fact that $a$ is a suitablyweighted measure of the number of degrees of freedom, which are irreversibly integrated out in the Wilsonian approach. In our case, the UV fixed is supposed to be interacting, and the IR fixed point is free. The constraints $a_{\mathrm{IR}}>0, b_{\mathrm{IR}}>0$ and $c_{\mathrm{IR}}>0$ are therefore automatically satisfied. There are, however, non-trivial constraints following from $b_{\mathrm{UV}}>0$ and $c_{\mathrm{UV}}>0$, and, assuming Cardy's conjecture is true, $\Delta a \equiv a_{\mathrm{UV}}-a_{\mathrm{IR}}>0$. The last condition is equivalent to

$$
\Delta a=\frac{1}{96} \operatorname{Tr}\left[\left(3 R_{i}-2\right)^{2}\left(3 R_{i}-5\right)\right]>0 .
$$

Note that the left side of this inequality has the opposite sign from that used in Ref. 20], since the correspondence between (UV, IR) and (free, interacting) fixed points has been switched.

In order to satisfy eq. (54), clearly at least one chiral superfield should have $R$-charge greater than $5 / 3$. This constraint, if it should really be imposed, is quite strong and would readily rule out UV-stable fixed points in many models. For example, consider SUSYQCD with $N_{f} \geq 3 N_{c}$ flavors. In that case, anomaly cancellation requires that $R_{Q}=R_{\bar{Q}}=1-N_{c} / N_{f}$. It follows that $a, b$, and $c$ are each positive. However, since $R_{Q}$ is not as large as 5/3, eq. (54) cannot be satisfied. Thus the rigorous exclusion or establishment of the existence of a UV-stable fixed point in this model would be a non-trivial test of Cardy's conjecture.

Let us assume that Cardy's conjecture is true, and explore under what circumstances a more complicated theory could satisfy it at a UV-stable fixed point. Even if a theory can be arranged to have a non-anomalous $R$ symmetry with one or more chiral superfields with $R_{i}>$ $5 / 3$, the rigorous constraint $b_{\mathrm{UV}}>0$ is then quite strong. For example, suppose that the theory includes chiral superfields $\Phi_{1}$ and $\Phi_{2}$ with the same gauge quantum numbers and $R_{1}, R_{2}>5 / 3$. Then there is a nonanomalous $U(1)$ flavor symmetry under which $\Phi_{1}$ and $\Phi_{2}$ have charges $F_{1}=1$ and $F_{2}=-1$ respectively and all other chiral superfields are neutral. This clearly implies the inconsistency $b_{\mathrm{UV}}<0$ for this flavor symmetry.

The simplest model that apparently can satisfy all constraints including eq. (54) is supersymmetric QCD with gauge group $S U\left(N_{c}\right), N_{f}$ flavors of quarks and antiquarks $Q+\bar{Q}$ in the fundamental + anti-fundamental representations, an adjoint $A$, and a singlet $S$. The anomaly cancellation condition for the $R$-charges is

$$
R_{Q}=R_{\bar{Q}}=1-\frac{N_{c}}{N_{f}} R_{A}
$$

Eq. (54) can only be satisfied if $R_{A}>5 / 3$. Now, if there were no superpotential, then there would be an anomaly-free $U(1)_{F}$ symmetry under which $(Q, \bar{Q}, A)$ have charges $\left(1,1,-N_{f} / N_{c}\right)$. The corresponding flavor symmetry central charge constraint $b_{\mathrm{UV}}>0$ would amount to

$$
2 N_{c} N_{f}\left(1-R_{Q}\right)+\left(1-\frac{1}{N_{c}^{2}}\right) N_{f}^{2}\left(1-R_{A}\right)>0,
$$

and this cannot be made consistent with eqs. (54) and (55). However, if we introduce a superpotential of the form

$$
W=S Q \bar{Q}+S^{3}
$$

then $U(1)_{F}$ is removed and there is no $b_{\mathrm{UV}}$ constraint. The superpotential eq. (57) also fixes the $R$-charges to have the unique values

$$
\begin{aligned}
& R_{S}=R_{Q}=R_{\bar{Q}}=2 / 3 ; \\
& R_{A}=\frac{N_{f}}{3 N_{c}} .
\end{aligned}
$$

Therefore one must have $N_{f}>5 N_{c}$ in order to have a UV-stable fixed point consistent with Cardy's conjecture. Writing $N_{f} / N_{c}=5+p$ with positive $p$, one finds 


$$
\begin{aligned}
\Delta a & =\left(N_{c}^{2}-1\right) \frac{(3+p)^{2} p}{96}>0 \\
c_{\mathrm{UV}} & =\frac{5+p}{96}\left[\left(N_{c}^{2}-1\right)\left(2+p+p^{2}\right)+8 N_{c}^{2}\right]>0,
\end{aligned}
$$

so all constraints are satisfied. The scaling dimensions of all gauge-invariant chiral superfields are at least 1 in this model. For example, the $R$-charges of gauge invariant chiral superfields are $R_{Q \bar{Q}}=4 / 3, R_{S}=2 / 3$, $R_{A^{2}}=2 N_{f} / 3 N_{c}$, leading to scaling dimensions 2,1 , and $N_{f} / N_{c}>5$ respectively.

A similar model consists of $S U\left(N_{c}\right)$ with $N_{f}$ flavors of quarks and antiquarks $Q, \bar{Q}$ and two adjoints $A_{1}$ and $A_{2}$. The superpotential at the putative fixed point is taken to be of the form

$$
W=A_{1} Q \bar{Q}+A_{1}^{3} .
$$

This fixes the anomaly-free $R$-charges to be

$$
\begin{aligned}
& R_{A_{1}}=R_{Q}=R_{\bar{Q}}=2 / 3 ; \\
& R_{A_{2}}=\frac{N_{f}+N_{c}}{3 N_{c}} .
\end{aligned}
$$

All of the constraints are satisfied for $N_{f}>4 N_{c}$ in this model.

As these examples illustrate, it is non-trivial to satisfy both $\Delta a>0$ and $b_{\mathrm{UV}}>0$ at a UV-stable fixed point. One must generally be attracted to a superpotential that eliminates dangerous flavor symmetries that could otherwise enter into the $b_{\mathrm{UV}}>0$ constraint, while allowing or enforcing $R_{i}>5 / 3$. Evidently, the RG flow into the UV will either not approach a fixed point, or will be attracted towards a special superpotential with the right properties. The superpotential that the theory is attracted towards in the UV may bear little resemblance to the IR superpotential.

Many more complicated examples can be constructed. For example, returning to the minimal missing partner $S U(5)$ model examined in section $\mathrm{\nabla}$, one finds that $\Delta a>0$ and $b_{\mathrm{UV}}>0$ for all flavor symmetries cannot simultaneously be achieved unless a superpotential is introduced. One way to proceed is to choose a superpotential that involves every chiral superfield except the adjoint, which then obtains an $R$-charge greater than $5 / 3$. In models with many higher-dimensional representations, it is often possible to find a superpotential which is a candidate for attraction to a UV-stable fixed point.

It should be emphasized that Cardy's constraint $\Delta a>$ 0 remains as a conjecture rather than a rigorously proved theorem. There are now a large number of examples [4,22,20] in which it is satisfied, and no other candidate for the 4-dimensional version of the $c$-theorem in 2 dimensions appears to acceptable. However, the known examples in which it has been checked are all free theories in the UV, and it is a possibility that it could be violated in theories with UV-stable fixed points. Therefore it remains an open question whether the constraint $\Delta a>0$ should necessarily be imposed. It seems an important challenge to see whether this can be made rigorous.

\section{CONCLUDING REMARKS}

We have investigated the hypothesis that a nonasymptotically free supersymmetric field theory flows to a fixed point in the ultraviolet. At one- and twoloop order, the gauge $\beta$ functions do not hint at this possibility, but fixed point indications do appear at three- and four-loop level. Although no perturbative calculation can prove the existence of a UV fixed point, the qualitative indications of higher-order calculations have inspired us to look more carefully at the possibility.

Starting with the fixed point assumption, the many exact results 23,24 of supersymmetric field theories enable us to investigate the consistency and implications of the claim. The most important rigorous consistency check is the requirement that all gauge-invariant and Lorentz invariant operators must have scaling dimension greater than or equal to 1 . This places a constraint on the slope of the gauge beta function, and ultimately on the strength of the power-law running for soft supersymmetry breaking parameters. The other important constraints arise from the positivity of trace anomaly coefficients, including the Cardy conjecture. These constraints, if indeed they are rigorously required, rule out some otherwise-consistent proposals for UV fixed point theories (e.g., simple SUSY QCD with $N_{f}>3 N_{c}$ ); however, they do not rule out the existence of UV fixed point theories in general, as we demonstrated by exhibiting viable examples.

The most important implication of a fixed point theory is the power-law running of the soft supersymmetry breaking masses. Going up in energy, the soft masses power-law run to small values in the UV fixed point region. For some applications, it is more convenient to think in the other direction. Namely, extremely small supersymmetry breaking parameters in the far UV power-law run to large (weak scale) values in the IR. This flow could generate a large hierarchy between the original supersymmetry breaking masses and the weak scale. If the flow is dominated by gaugino masses, the scalar masses will be flavor-blind due to the running near the superconformal UV-stable fixed point, yielding a solution to the supersymmetric flavor problem. (In some sense, this is an opposite scenario to that of [25], which invokes an infrared-stable fixed point to solve the SUSY flavor problem.)

We note that near the fixed point, non-renormalizable 
operators may become relevant due to large anomalous dimensions. For superpotential operators, this seems unlikely since the anomalous dimensions are typically positive. However, we have no such control over Kähler potential terms. Therefore, a true solution to the supersymmetric flavor problem requires the (not unreasonable, we believe) assumption that these are still subdominant compared to contributions from power-law running of flavor-blind gaugino masses.

Such a large hierarchy may also have implications for the gravitino problem [26], since the ultimate supersymmetry-breaking order parameter $F$ could actually be much smaller than the power-law-enhanced masses of the MSSM superpartners. Also, if the gravitino is light enough, superpartners could decay into it promptly, leading to collider signatures characteristic of gauge mediation [27]: e.g. hard photons plus missing energy, or hard leptons plus missing energy. Because of the constraint on the anomalous dimension of gaugino masses discussed in section IV, this would require that the theory remains near the UV-stable fixed point over a range of $\mathrm{RG}$ scales corresponding to $\Delta t$ significantly larger than $\ln \left(M_{\mathrm{GUT}} / M_{\mathrm{Planck}}\right)$.

It may be difficult to uniquely identify a superpartner mass spectrum as arising from an ultraviolet fixed point. The $S U(5)$ GUT missing partner model example that we briefly considered in the text might look qualitatively like any other theory with unified gaugino masses. However, it is also quite possible that the power-law running in the UV at very high scales would generate a different scalar superpartner spectrum than the ones from gauge mediation or the usual assumptions of minimal supergravity, and the ideas could in principle be resolved. To do this in detail would require an improved understanding of the non-perturbative running of soft scalar masses near the fixed point.

Acknowledgments: We are grateful to N. Arkani-Hamed, W. Bardeen and M. Shifman for helpful conversations. This work was supported in part by National Science Foundation Grant No. PHY-9970691 and by the Alfred P. Sloan Foundation.

[1] H. E. Haber and G. L. Kane, "The Search For Supersymmetry: Probing Physics Beyond The Standard Model," Phys. Rept. 117, 75 (1985); S.P. Martin "A supersymmetry primer", hep-ph/9709356.

[2] M. Dine and N. Seiberg, "Is the superstring weakly coupled?," Phys. Lett. B162, 299 (1985); T. Banks and M. Dine, "Coping with strongly coupled string theory,"
Phys. Rev. D50, 7454 (1994) hep-th/9406132.

[3] K. S. Babu and J. C. Pati, "The problems of unification mismatch and low alpha(3): A solution with light vector-like matter," Phys. Lett. B384, 140 (1996) hepph/9606215; C. Kolda and J. March-Russell, "Lowenergy signatures of semi-perturbative unification," Phys. Rev. D55, 4252 (1997) hep-ph/9609480; D. Ghilencea, M. Lanzagorta and G. G. Ross, "Strong unification," Phys. Lett. B415, 253 (1997) hep-ph/9707462.

[4] J.L. Cardy, "Is there a C theorem in four-dimensions?," Phys. Lett. B215, 749 (1988).

[5] A. B. Zamolodchikov, "'Irreversibility' Of The Flux Of The Renormalization group in a 2-D field theory," JETP Lett. 43, 730 (1986).

[6] J. Polchinski, "Scale and conformal invariance in quantum field theory," Nucl. Phys. B303, 226 (1988). See also L. O'Raifeartaigh, I. Sachs and C. Wiesendanger, "Weyl gauging and conformal invariance," Nucl. Phys. B495, 433 (1997) hep-th/9607110.

[7] V. K. Dobrev and V. B. Petkova, "All positive energy unitary irreducible representations of extended conformal supersymmetry," Phys. Lett. B162, 127 (1985); S. Minwalla, "Restrictions imposed by superconformal invariance on quantum field theories," Adv. Theor. Math. Phys. 2, 781 (1998) hep-th/9712074].

[8] N. Seiberg, "Electric-magnetic duality in supersymmetric non-abelian gauge theories," Nucl. Phys. B435, 129 (1995) hep-th/9411149.

[9] I. I. Kogan, M. Shifman and A. Vainshtein, "Matching conditions and duality in N=1 SUSY gauge theories in the conformal window," Phys. Rev. D53, 4526 (1996) hep-th/9507170.

[10] T. Banks and A. Zaks, "On the phase structure of vectorlike gauge theories with massless fermions," Nucl. Phys. B196, 189 (1982).

[11] R. G. Leigh and M. J. Strassler, "Exactly marginal operators and duality in four-dimensional $\mathrm{N}=1$ supersymmetric gauge theory," Nucl. Phys. B447, 95 (1995) hep-th/9503121.

[12] J. Hisano and M. Shifman, "Exact results for soft supersymmetry breaking parameters in supersymmetric gauge theories," Phys. Rev. D56, 5475 (1997) hepph/9705417; I. Jack and D.R.T. Jones, "The gaugino beta-function," Phys. Lett. B415, 383 (1997) hepph/9709364]; I. Jack, D.R.T. Jones and A. Pickering, "Renormalisation invariance and the soft beta functions," Phys. Lett. B426, 73 (1998) hep-ph/9712542.

[13] G. Mack, "All unitary ray representations of the conformal group $S U(2,2)$ with positive energy," Commun. Math. Phys. 55, 1 (1977).

[14] I. Jack, D.R.T. Jones and A. Pickering, "The soft scalar mass beta-function," Phys. Lett. B432, 114 (1998) hepph/9803405; A. Karch, T. Kobayashi, J. Kubo and G. Zoupanos, "Infrared behavior of softly broken SQCD and its dual," Phys. Lett. B441, 235 (1998) hhepth/9808178.

[15] I. Jack, D.R.T. Jones and C.G. North, " $N=1$ supersymmetry and the three loop anomalous dimension for the chiral superfield," Nucl. Phys. B473, 308 (1996) hep-ph/9603386; "Scheme dependence and the NSVZ beta-function," Nucl. Phys. B486, 479 (1997) thep- 
ph/9609325]. "N=1 supersymmetry and the three loop gauge beta function," Phys. Lett. B386, 138 (1996) hepph/9606323; I. Jack, D.R.T. Jones and A. Pickering, "The connection between the DRED and NSVZ renormalisation schemes," Phys. Lett. B435, 61 (1998) hepph/9805482.

[16] W. Siegel, "Supersymmetric dimensional regularization via dimensional reduction," Phys. Lett. B84, 193 (1979); D.M. Capper, D.R.T. Jones and P. van Nieuwenhuizen, "Regularization by dimensional reduction of supersymmetric and nonsupersymmetric gauge theories," Nucl. Phys. B167, 479 (1980).

[17] V.A. Novikov, M.A. Shifman, A.I. Vainshtein and V.I. Zakharov, "Exact Gell-Mann-Low function of supersymmetric Yang-Mills theories from instanton calculus," Nucl. Phys. B229, 381 (1983); "Supersymmetric instanton calculus: Gauge theories with matter," Nucl. Phys. B260, 157 (1985); "Beta function in supersymmetric gauge theories: instantons versus traditional approach," Phys. Lett. B166, 329 (1986).

[18] A. Masiero, D. V. Nanopoulos, K. Tamvakis and T. Yanagida, "Naturally massless Higgs doublets in supersymmetric SU(5)," Phys. Lett. B115, 380 (1982); B. Grinstein, "A Supersymmetric $S U(5)$ gauge theory with no gauge hierarchy problem," Nucl. Phys. B206, 387 (1982).

[19] P.M. Ferreira, I. Jack and D.R.T. Jones, "LargeN supersymmetric beta-functions," Phys. Lett. B399, 258 (1997) hep-ph/9702304; P.M. Ferreira, I. Jack, D.R.T. Jones and C.G. North, "Beta-functions in large$N(f)$ supersymmetric gauge theories," Nucl. Phys. B504, 108 (1997) hep-ph/9705328; I. Jack and D.R.T. Jones, "Regularisation of supersymmetric theories," hep-ph/9707278.

[20] D. Anselmi, D. Z. Freedman, M. T. Grisaru and A. A. Johansen, "Nonperturbative formulas for central functions of supersymmetric gauge theories," Nucl. Phys. B526, 543 (1998) hep-th/9708042; D. Anselmi, J. Erlich, D. Z. Freedman and A. A. Johansen, "Positivity constraints on anomalies in supersymmetric gauge theories," Phys. Rev. D57, 7570 (1998) hep-th/9711035;

[21] D. Anselmi, "Central functions and their physical implications," JHEP 9805, 005 (1998) hep-th/9702056.

[22] F. Bastianelli, "Tests for C-theorems in 4D," Phys. Lett. B369, 249 (1996) hep-th/9511065.

[23] M. Shifman, "Nonperturbative dynamics in supersymmetric gauge theories," Prog. Part. Nucl. Phys. 39, 1 (1997) hep-th/9704114.

[24] N. Arkani-Hamed and H. Murayama, "Holomorphy, rescaling anomalies and exact beta functions in supersymmetric gauge theories," JHEP 0006, 030 (2000) hep-th/9707133; N. Arkani-Hamed, G. F. Giudice, M. A. Luty and R. Rattazzi, "Supersymmetry-breaking loops from analytic continuation into superspace," Phys. Rev. D58, 115005 (1998) hep-ph/9803290; N. ArkaniHamed and R. Rattazzi, "Exact results for nonholomorphic masses in softly broken supersymmetric gauge theories," Phys. Lett. B454, 290 (1999) hepth/9804068.

[25] A.E. Nelson and M.J. Strassler, "Suppressing flavor anarchy," hep-ph/0006251.
[26] H. Pagels and J. R. Primack, "Supersymmetry, cosmology and new TeV physics," Phys. Rev. Lett. 48, 223 (1982); S. Weinberg, "Cosmological constraints on the scale of supersymmetry breaking," Phys. Rev. Lett. 48, 1303 (1982); J. Ellis, J. E. Kim and D. V. Nanopoulos, "Cosmological gravitino regeneration and decay," Phys. Lett. B145, 181 (1984); M. Kawasaki and T. Moroi, "Gravitino production in the inflationary universe and the effects on big bang nucleosynthesis," Prog. Theor. Phys. 93, 879 (1995) hep-ph/9403364.

[27] G. F. Giudice and R. Rattazzi, "Theories with gaugemediated supersymmetry breaking," Phys. Rept. 322, 419 (1999) hep-ph/9801271]; R. Culbertson et al., "Lowscale and gauge-mediated supersymmetry breaking at the Fermilab Tevatron Run II," hep-ph/0008070. 